\title{
Application of Reduced-FOV Diffusion-Weighted Imaging in Evaluation of Normal Pituitary Glands and Pituitary Macroadenomas
}

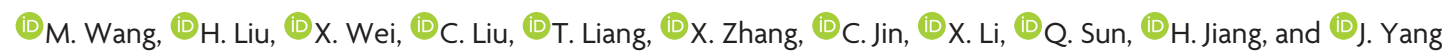 \\ O- E E
}

\begin{abstract}
BACKGROUND AND PURPOSE: FOV optimized and constrained undistorted single-shot imaging provides relatively high-resolution images with few artifacts. This study evaluated the image quality and value of FOV optimized and constrained undistorted single-shot DWI in the evaluation of normal pituitary glands and pituitary macroadenomas.
\end{abstract}

MATERIALS AND METHODS: Subjects with normal pituitary glands and patients with pituitary macroadenomas underwent FOV optimized and constrained undistorted single-shot and EPI DWI. Two neuroradiologists graded the image quality based on visualization of the pituitary stalk, pituitary gland, and pituitary macroadenoma. Intra- and interobserver agreements were assessed by $\kappa$ statistics. Image quality and ADCs were compared between the 2 methods by the paired Wilcoxon signed rank test and $t$ test. Differences in ADC between normal pituitary glands and macroadenomas were analyzed by the independent-samples $t$ test.

RESULTS: Twenty-eight subjects with normal pituitary glands and 16 patients with macroadenomas were enrolled. Intra- and interobserver agreements for image-quality assessment were moderate to substantial. Relative to EPI DWI, FOV optimized and constrained undistorted single-shot DWI exhibited obviously better image quality both in normal pituitary glands and macroadenomas. There was no significant difference in ADCs of macroadenomas between the 2 methods. Macroadenomas with soft consistency $\left(0.75 \pm 0.14 \times 10^{-3}\right.$ $\left.\mathrm{mm}^{2} / \mathrm{s}\right)$ had significantly lower mean ADC than normal pituitary glands $\left(1.18 \pm 0.19 \times 10^{-3} \mathrm{~mm}^{2} / \mathrm{s} ; P<.001\right)$.

CONCLUSIONS: FOV optimized and constrained undistorted single-shot DWI helps acquire high-resolution images of normal pituitary glands and pituitary macroadenomas with relatively few susceptibility artifacts in a clinically feasible scan time. This sequence might be helpful for evaluating the consistency of pituitary macroadenomas.

ABBREVIATION: FOCUS $=$ FOV optimized and constrained undistorted single-shot

D iffusion-weighted imaging can not only help detect pituitary apoplexy and differentiate pituitary mass lesions but can also help assess the consistency of pituitary macroadenomas, evaluate the diffusivity of normal pituitary glands, and predict the success of surgical treatment. ${ }^{1-5}$ It contributes to deciding the best surgical strategy and reducing postoperative complications. The most

Received August 31, 2017; accepted after revision April 13, 2018.

From the Department of Diagnostic Radiology (M.M.W., H.L., X.C.W., C.C.L., T.L., X.H.Z., C.J., X.J.L., Q.L.S., H.X.J., J.Y.), the First Affiliated Hospital, and Department of Biomedical Engineering (H.L., T.L., Q.L.S., H.X.J., J.Y.), the Key Laboratory of Biomedical Information Engineering of the Ministry of Education, School of Life Science and Technology, Xi'an Jiaotong University, Xi'an, China.

This work was supported by a grant from the National Natural Science Foundation of China (No. 81171317, 81471631, 81771810), the National Key Research and Development Program of China (2016YFC0100300), the 2011 New Century Excellent Talent Support Plan from the Ministry of Education of China (NCET-11-0438), and the Clinical Research Award of the First Affiliated Hospital of Xi'an Jiaotong University (XJTUIAF-CRF-2015-004).

Please address correspondence to Jian Yang, PhD, Department of Diagnostic Radiology, the First Affiliated Hospital, Xi'an Jiaotong University, Xi'an, Shaanxi, China; e-mail: yj1118@mail.xjtu.edu.cn widely used DWI technique is EPI DWI, which has limitations such as low spatial resolution, strong susceptibility artifacts, and distortion due to eddy currents, especially in the sellar region. ${ }^{6}$ Consequently, this sequence has limited application and can provide valuable information only for large tumors. Previous studies have attempted to use various diffusion sequences, such as line scan, ${ }^{7}$ readout segmentation of long variable echo trains, ${ }^{8}$ BLADE (Siemens, Erlangen, Germany), and PROPELLER, ${ }^{5,9-11}$ to overcome issues related to artifacts and image degradation and to analyze the diffusivities of tumors. However, at present, there is only experiential knowledge regarding the relationship between normal pituitary glands and pituitary adenomas, which shows that pituitary adenomas are often soft and white in contrast to the more firm, orange-yellow anterior pituitary

\footnotetext{
- Indicates open access to non-subscribers at www.ajnr.org

$\equiv$ Indicates article with supplemental on-line table.

Indicates article with supplemental on-line photo.

http://dx.doi.org/10.3174/ajnr.A5735
} 
tissue. ${ }^{12}$ Because of inadequate image quality in the sellar region, the diffusion characteristics of these 2 tissues have not been fully elucidated.

Recently, FOV optimized and constrained undistorted singleshot (FOCUS; GE Healthcare, Milwaukee, Wisconsin) DWI has been reported to have superior performance in the evaluation of the spine, ${ }^{13}$ neck tumors, ${ }^{14}$ pancreas, ${ }^{15}$ breast, ${ }^{16}$ and prostate. ${ }^{17}$ This improved small-FOV sequence uses a $90^{\circ} 2 \mathrm{D}$ spatially selective radiofrequency pulse and a $180^{\circ}$ refocusing pulse. The $2 \mathrm{D}$ radiofrequency pulse excites only a limited extent of tissue along the phase-encoding direction in the target slice, resulting in a multislice and restrained FOV excitation without introducing aliasing artifacts. This technique not only enables a reduced number of $k$-space lines in the phase-encoding direction but also provides a higher resolution for a fixed scan time. Additionally, the reduced FOV decreases the readout duration needed for imaging to reduce off-resonance-induced artifacts. ${ }^{13,18}$ To the best of our knowledge, the application of FOCUS DWI for the evaluation of normal pituitary glands and pituitary macroadenomas has not been reported to date. The purpose of this study was to evaluate the image quality and value of FOCUS DWI in the evaluation of normal pituitary glands and pituitary macroadenomas.

\section{MATERIALS AND METHODS}

This study was conducted with approval from the involved institutional review boards of the First Affiliated Hospital of Xi'an Jiaotong University.

\section{Subjects}

Between December 2016 and January 2018, subjects with normal pituitary glands and patients with preoperative pituitary macroadenomas were enrolled in this study. All participants were imaged by FOCUS and EPI DWI in succession. Subjects in the normal pituitary gland group who underwent brain MR imaging examinations for the evaluation of headache or dizziness were eligible for inclusion if they met the following conditions: 1) at least 20 years of age; 2) no abnormalities of the pituitary gland on conventional MR imaging and DWI sequences; 3) no history of pituitary, sellar, or hypothalamus diseases; 4) no history of endocrine medication; 5) no suspected or diagnosed hormonal imbalance within 1 year; and 6) no history of central nervous system tumor, skull trauma, operation, radiation, or chemotherapy. The exclusion criteria were as follows: 1) breast diseases, 2) lung cancer, and 3) artifacts caused by oral implants. Patients with pituitary macroadenomas were enrolled in accordance with the following criteria: 1) lesion size $>10 \mathrm{~mm}$ on MR images, and 2) diagnosis confirmed by pathologic and immunohistochemical examination. Patients with any preoperative treatment were excluded, as were subjects with incomplete MR imaging data.

\section{MR Imaging}

All MR images were acquired using a 3T system (Discovery 750w; GE Healthcare) with a 24-channel head coil. Pituitary MR imaging was performed using the following routine sequences: sagittal and coronal T1WI $(\mathrm{TR} / \mathrm{TE}=400 \mathrm{~ms} / \mathrm{minimum}, \mathrm{FOV}=20 \mathrm{~cm}$,
Table 1: Imaging parameters for DWI sequences

\begin{tabular}{lcc} 
& FOCUS DWI & EPI DWI \\
\hline TR $(\mathrm{ms})$ & 2200 & 2200 \\
TE $(\mathrm{ms})$ & Minimum & Minimum \\
B-value $\left(\mathrm{s} / \mathrm{mm}^{2}\right)$ & 0,500 & 0,500 \\
Diffusion directions & All & All \\
Frequency-encoding direction & $\mathrm{S} / \mathrm{I}$ & $\mathrm{S} / \mathrm{I}$ \\
FOV $(\mathrm{cm})$ & $16 \times 4.8$ & $24 \times 24$ \\
Matrix size & $128 \times 38$ & $160 \times 160$ \\
NEX for $\mathrm{B}_{0}$ & 4 & 4 \\
NEX for $b=500$ & 12 & 12 \\
Slice thickness $(\mathrm{mm})$ & 2.0 & 2.0 \\
Intersection gap $(\mathrm{mm})$ & 0 & 0 \\
Spatial resolution $\left.(\mathrm{mm})^{3}\right)$ & $1.25 \times 1.26 \times 2$ & $1.5 \times 1.5 \times 2$ \\
Acquisition time $(\mathrm{min}: \mathrm{s})$ & $01: 30$ & $01: 30$ \\
\hline
\end{tabular}

Note:-S/I indicates superior/inferior.

slice thickness $=2 \mathrm{~mm}$ without intersection gap) and sagittal and coronal T2WI $(\mathrm{TR} / \mathrm{TE}=3880 / 128 \mathrm{~ms}, \mathrm{FOV}=18 \mathrm{~cm}$, slice thickness $=2 \mathrm{~mm}$ without intersection gap). In 12 patients, enhanced coronal T1WI was acquired after injection of $0.2 \mathrm{mmol} / \mathrm{kg}$ of contrast medium (Gd-DTPA, Magnevist; Bayer HealthCare Pharmaceuticals, Wayne, New Jersey). Before Gd-DTPA administration, FOCUS and EPI DWI were acquired in the coronal plane. Imageacquisition parameters are presented in Table 1.

\section{Image Analysis}

Assessment of Image Quality. The MR images were anonymized and reviewed with the same window widths and levels on the same workstation. Two experienced neuroradiologists independently reviewed coronal images ( $b=500 \mathrm{~s} / \mathrm{mm}^{2}$ ) acquired using FOCUS and EPI sequences and evaluated their image quality with reference to coronal T1WI. On the basis of the degrees of susceptibility artifacts and spatial distortion as well as the quality of anatomic details displayed, image quality was scored from 0 to 4 for normal pituitary glands and 0 to 5 for pituitary macroadenomas (Table 2). Two months after initial review, the MR images were reviewed again by one of the observers.

Measurement of ADC Values. All image analyses for ADC mapping were performed on the ADW4.6 workstation (GE Healthcare). For ADC calculation, ROIs were drawn on MR images $\left(b=500 \mathrm{~s} / \mathrm{mm}^{2}\right)$ by one of the observers and cross-referenced with the anatomic structures on T1WI. In the normal pituitary gland group, ROIs $\left(10-15 \mathrm{~mm}^{2}\right)$ were placed on the anterior lobe of the pituitary gland. In the macroadenoma group, ROIs $\left(20-30 \mathrm{~mm}^{2}\right)$ were placed on regions with a solid appearance, taking care to avoid cysts, hemorrhages, blood vessels, and areas with visible image distortion/signal loss. To minimize bias, we measured each area 3 times and calculated the average ADCs.

\section{Statistical Analysis}

Statistical analysis was performed using SPSS 18.0 (IBM, Armonk, New York). The Wilcoxon signed rank test and $\chi^{2}$ test were performed to compare the age and sex between the normal pituitary gland and macroadenoma groups. Intra- and interobserver agreements were assessed using $\kappa$ statistics, with the level of agreement indicated as follows: poor, $\kappa=0.00-0.20$; slight, $\kappa=0.21-0.40$; moderate, $\kappa=0.41-0.60$; substantial, $\kappa=0.61-0.80$; and almost perfect, $\kappa=0.81-1.00$. Disagreements regarding imaging find- 
ings were resolved by discussion and agreement. The paired Wilcoxon signed rank test and $t$ test were used for comparison of image quality and ADCs between FOCUS and EPI DWI. Differences in ADCs between normal pituitary glands and macroadenomas were analyzed using the independent-samples $t$ test. $P<.05$ was considered statistically significant.

\section{RESULTS}

Of 56 participants (34 subjects in the normal pituitary gland group and 22 patients in the macroadenoma group) reviewed, 28 subjects with normal pituitary glands ( 11 men and 17 women; age range, 22-79 years; median age, 40 years) and 16 patients with macroadenomas ( 8 men and 8 women; age range, $20-73$ years; median age, 48 years) were included. In the normal pituitary

Table 2: Evaluation indexes of DWI

\begin{tabular}{|c|c|}
\hline Score & Criteria \\
\hline \multicolumn{2}{|c|}{ Normal pituitary gland } \\
\hline 0 & $\begin{array}{l}\text { Pronounced artifacts; pituitary stalk and gland cannot be } \\
\text { recognized }\end{array}$ \\
\hline 1 & $\begin{array}{l}\text { Considerable artifacts; the stalk is visible, but the gland } \\
\text { cannot be recognized }\end{array}$ \\
\hline 2 & $\begin{array}{l}\text { Pituitary stalk and gland are visible, with moderate-to- } \\
\text { obvious image distortion and/or most of the pituitary } \\
\text { gland ( }>50 \%) \text { exhibiting signal loss }\end{array}$ \\
\hline 3 & $\begin{array}{l}\text { Pituitary stalk and gland are distinctly visible, with mild } \\
\text { image distortion and/or }<50 \% \text { of the pituitary } \\
\text { gland exhibiting signal loss }\end{array}$ \\
\hline 4 & $\begin{array}{l}\text { Visualization of pituitary stalk and gland is as clear as } \\
\text { that on TIWI }\end{array}$ \\
\hline \multicolumn{2}{|c|}{ Pituitary macroadenoma } \\
\hline 0 & Pronounced artifacts; adenoma cannot be recognized \\
\hline 1 & $\begin{array}{l}\text { Considerable artifacts; adenoma is visible, with or without } \\
\text { most of the adenoma (>50\%) exhibiting signal loss }\end{array}$ \\
\hline 2 & $\begin{array}{l}\text { Adenoma is visible, with obvious image distortion and with } \\
\text { or without nearly half of the adenoma }(25 \%-50 \%) \\
\text { exhibiting signal loss }\end{array}$ \\
\hline 3 & $\begin{array}{l}\text { Adenoma is distinctly visible; moderate image distortion, } \\
\text { with or without }<25 \% \text { of the adenoma exhibiting signal } \\
\text { loss }\end{array}$ \\
\hline 4 & $\begin{array}{l}\text { Adenoma is distinctly visible, with only mild image } \\
\text { distortion }\end{array}$ \\
\hline 5 & Visualization of adenoma is as clear as that on TIWI \\
\hline
\end{tabular}



T1WI

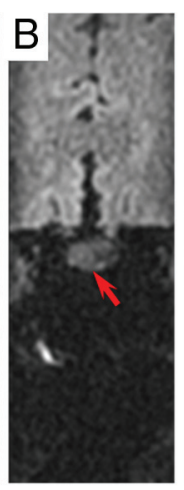

FOCUS DWI

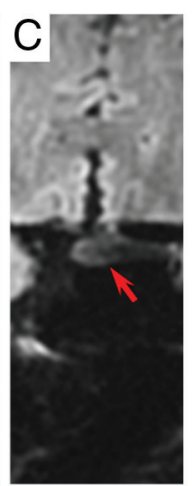

EPI DWI

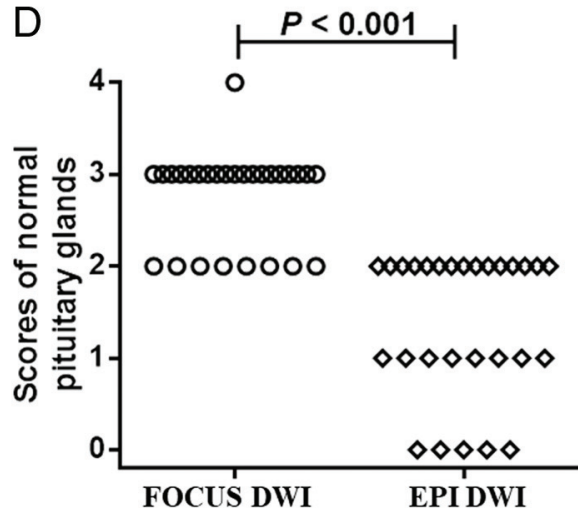

FOCUS DWI gland group, 6 subjects were excluded with breast diseases $(n=1)$, lung cancer $(n=3)$, and artifacts caused by oral implants $(n=2)$. Six patients in the macroadenoma group with preoperative treatment $(n=4)$ and incomplete MR imaging data $(n=2)$ were also excluded. There were no significant differences in age $(P=.15)$ and $\operatorname{sex}(P=.49)$ between the 2 groups. Among the 16 patients, 13 had nonfunctional macroadenomas, 2 had prolactin-producing macroadenomas, and 1 patient had a growth-hormone-producing macroadenoma. All tumors were amenable to resection with the direct endoscopic transsphenoidal technique, and an operation of nonfunctional macroadenomas was undergone due to compression symptoms. With H\&E staining of the specimens, the extracellular matrix (mainly composed of collagen) of each tumor was found to account for $<5 \%$ of the entire area of stained tissue; consequently, all macroadenomas were considered of soft consistency.

\section{Comparison of Image Quality between FOCUS and EPI DWI}

The intraobserver agreement for image quality was substantial for both normal pituitary glands $(\kappa=0.79, P<.001)$ and pituitary macroadenomas $(\kappa=0.74$, $P<.001$ ). The interobserver agreement was moderate to substantial for both normal pituitary glands $(\kappa=0.61, P<$ $.001)$ and pituitary macroadenomas $(\kappa$ $=0.57, P<.001)$.

The image-quality scores of the 2 sequences are presented in Figs 1 and 2. Images acquired using the FOCUS sequence showed no or minimal degrees of artifacts and distortion, a finding significantly better than the performance of EPI in both normal pituitary glands (median score: 3 versus 2; $P<.001$ ) and macroadenomas (median score: 4 versus $3 ; P<.001)$.

\section{Measurement of ADC in Normal Pituitary Glands and Pituitary Macroadenomas}

While FOCUS DWI provided images without obvious distortion in 24 subjects with normal pituitary glands and all patients, images acquired by EPI DWI failed ADC measurement in 25 subjects in the normal pituitary gland group and 1 patient in the macroadenoma group because of unidentifiable anatomic structures caused by severe image degradation and artifacts. In FOCUS DWI, the mean ADC of the anterior lobe of normal pituitary glands was $1.18 \pm$ $0.19 \times 10^{-3} \mathrm{~mm}^{2} / \mathrm{s}$ (range, $0.83 \times 10^{-3}$ to $\left.1.49 \times 10^{-3} \mathrm{~mm}^{2} / \mathrm{s}\right)$. In pituitary macroadenomas, ADCs measured in FOCUS and EPI DWI were $0.75 \pm$

-C Images of a 68-year-old woman with dizziness for 1 week. The pituitary gland score of 3 for FOCUS DWI and 2 for EPI DWI, respectively (red arrow indicates the pituitary gland). The mean ADC value of the FOCUS DWI measurement is $1.19 \times 10^{-3} \mathrm{~mm}^{2} / \mathrm{s}$. $D$, The image-quality scores of normal pituitary glands between the 2 methods are statistically significant $(P<.001)$. The number of subjects for each score in FOCUS and EPI DWI sequences is shown in the On-line Table. 


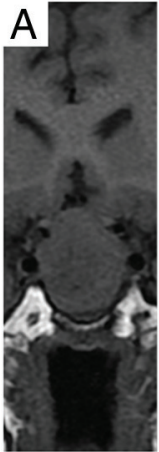

T1WI

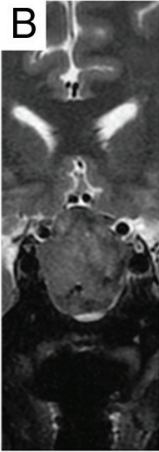

T2WI

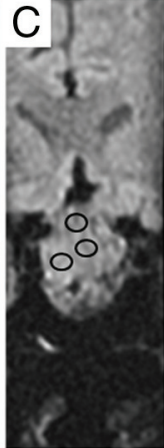

FOCUS DWI EPI DWI

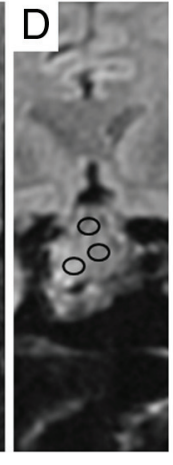

EPIDWI

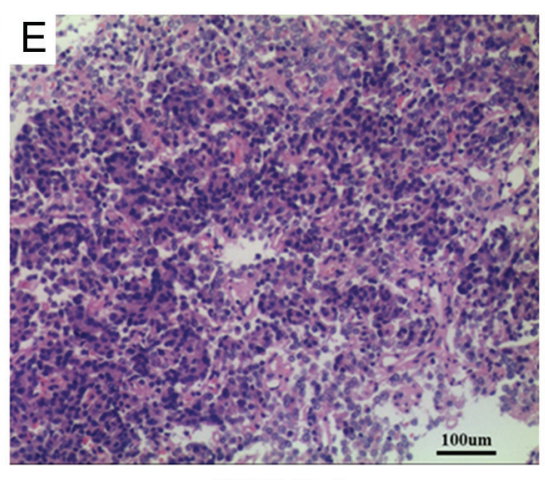

H\&E Stain

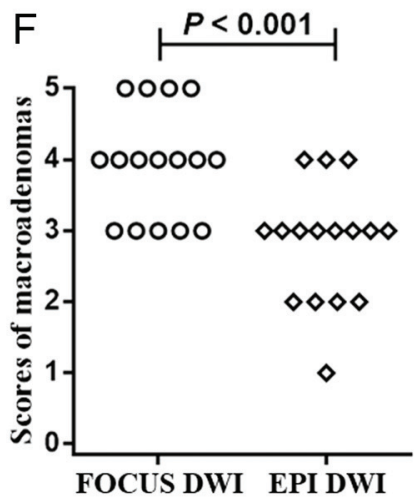

FIG 2. $A-D$, Images of a 51-year-old man with immunohistochemical staining-proved nonfunctional adenoma. The scores of the macroadenoma in FOCUS and EPI DWI are 4 and 3, respectively (black circles indicate the ROls of ADC measurements). The mean ADC values of the FOCUS and EPI DWI measurements are $0.66 \times 10^{-3} \mathrm{~mm}^{2} / \mathrm{s}$ and $0.80 \times 10^{-3} \mathrm{~mm}^{2} / \mathrm{s}$, respectively. E, A specimen of the mass at histologic examination (H\&E stain; original magnification, $\times 200$ ) shows plenty of small cells (blue) with scant fibrous stroma (deep pink). $F$, The image-quality scores of macroadenomas between 2 methods are statistically significant $(P<.001)$. The number of patients for each score in FOCUS and EPI DWI sequences is shown in the On-line Table.

$0.14 \times 10^{-3} \mathrm{~mm}^{2} / \mathrm{s}$ (range, $0.54 \times 10^{-3}$ to $0.99 \times 10^{-3} \mathrm{~mm}^{2} / \mathrm{s}$ ) and $0.77 \pm 0.18 \times 10^{-3} \mathrm{~mm}^{2} / \mathrm{s}$ (range, $0.53 \times 10^{-3}$ to $1.11 \times$ $10^{-3} \mathrm{~mm}^{2} / \mathrm{s}$ ), respectively. There was no significant difference in mean tumor ADC between the 2 methods $(P=.45)$. The ADC measurements of the 2 methods based on individuals are shown in the On-line Figure. Furthermore, in the FOCUS DWI, the mean $\mathrm{ADC}$ of the pituitary macroadenomas $\left(0.75 \pm 0.14 \times 10^{-3}\right.$ $\mathrm{mm}^{2} / \mathrm{s}$ ) was significantly lower than that of the anterior lobe of the normal pituitary glands $\left(1.18 \pm 0.19 \times 10^{-3} \mathrm{~mm}^{2} / \mathrm{s} ; P<\right.$ $.001)$.

\section{DISCUSSION}

This study demonstrated that FOCUS DWI enables acquisition of high-resolution images of both normal pituitary glands and pituitary macroadenomas with fewer artifacts and less distortion than EPI DWI. In terms of quantitative findings, although both imaging sequences provided generally comparable ADCs of macroadenomas, the diffusivity distribution of macroadenomas with soft consistency was lower than that of the anterior lobe of normal pituitary glands in the present study population.

Conventional single-shot EPI is the most commonly used DWI technique in routine clinical practice. However, because of its vulnerability to the off-resonance effect, it has a severely limited capability for imaging the sellar region. Because the pituitary gland is surrounded by the sphenoid sinus and cavernous sinuses, the presence of a series of air-tissue interfaces in this area alters the local magnetic field, resulting in $\mathrm{B}_{0}$-related artifacts. ${ }^{3,11}$ The presence of heterogeneities finally leads to signal pile-up and image distortion and renders small lesions and normal structures unrecognizable, especially in case of structures located near the skull base. ${ }^{11}$ Taking all these factors into account, the present study used semiquantitative indexes for qualitative assessment, which proved to be highly feasible and exhibited good repeatability. In the present study, EPI DWI showed greater degrees of obvious artifacts, geometric distortion, and signal loss than FOCUS DWI. All these factors serve to severely degrade the quality of overall visualization of anatomic structures and abnormalities, thus decreasing the accuracy of ADC measurement as well as the value of an auxiliary diagnosis. Both imaging protocols in the present study used the same TRs, b-values, slice thicknesses, and NEX, but not the same FOV. This is because a FOV of $24 \mathrm{~cm}$ for EPI DWI can include all tissues along the phase-encoding direction, which aims to avoid aliasing artifacts. For FOCUS DWI, there are 2 reasons for us to choose $16 \mathrm{~cm}$ instead of $24 \mathrm{~cm}$ in the frequencyencoding direction. First, a smaller FOV is achievable for FOCUS but not for EPI, which makes higher spatial resolution easier. Second, it takes fewer steps to encode a smaller FOV, and this would further benefit the image-distortion control. As a result, only FOCUS DWI demonstrated the ability to acquire images of high spatial resolution and less distortion, while maintaining a clinically feasible scan time.

On DWI, differences in signal intensity and contrast between different tissues depend on the b-values. To date, no study has suggested the optimal b-value for evaluation of the normal pituitary gland and pituitary diseases. It is common knowledge that MR signal intensity and the SNR of DWI decrease with an increase in b-values. ${ }^{19}$ Previous pituitary studies mainly used a b-value of $1000 \mathrm{~s} / \mathrm{mm}^{2}$ and a slice thickness ranging from 3 to $6 \mathrm{~mm}$, with varying degrees of intersection gap. ${ }^{3,7,10,20}$ After a careful tradeoff among imaging parameters such as b-value, slice thickness, and spatial resolution, we finally chose a b-value of $500 \mathrm{~s} / \mathrm{mm}^{2}$ for FOCUS DWI in the present study.

Among the DWI techniques that can reduce the susceptibility artifacts of the sellar region, the scanning time for FOCUS DWI (1 minute 30 seconds) is shorter than those for line scan ( 1 minute 57 seconds to 3 minutes 15 seconds $)^{7}$ and the readout segmentation of long variable echo trains ( 3 minutes 28 seconds $)^{8}$ in similar slice thicknesses and/or in-plane resolutions. For the advanced 3D diffusion-sensitized driven-equilibrium turbo field-echo sequence (5 minutes 22 seconds) ${ }^{4}$ and BLADE/PROPELLER (5 minutes 51 seconds), ${ }^{11}$ a relatively long acquisition time is needed to significantly reduce and even remove the susceptibility-related signal loss and image distortion. Their scanning time is $>3$ times longer than that of FOCUS DWI. Thus, FOCUS DWI can acquire high-quality images in a relatively short time, which not only 
boosts patient throughput but also minimizes the possibility of patient motion during scanning.

The mean ADC of the anterior lobe of the normal pituitary glands in the present study $\left(1.18 \pm 0.19 \times 10^{-3} \mathrm{~mm}^{2} / \mathrm{s}\right)$ is in agreement with the values reported by Hiwatashi et al. ${ }^{4}$ As speculated by these authors, lack of myelination and hypervascularity might contribute to diffusivity. Despite the resolution of FOCUS DWI $\left(1.25 \times 1.26 \times 2 \mathrm{~mm}^{3}\right)$ being slightly higher than that reported for diffusion-sensitized driven-equilibrium turbo field echo imaging $\left(1.5 \times 1.5 \times 1.5 \mathrm{~mm}^{3}\right),{ }^{4}$ it is still difficult to evaluate the diffusivity of the posterior lobe of the pituitary gland. In addition, in the present study, the difference between average ADC values of the 2 sequences in the soft macroadenoma group was not significant. This finding is consistent with the results reported by studies on the spinal cord, ${ }^{21}$ prostate cancer, ${ }^{17}$ and the pancreas. ${ }^{15}$ In contrast, some studies on breast cancer have found that the lower ADC of reduced-FOV DWI relative to that of EPI DWI is attributable to the decreased partial volume effect between tumors and surrounding healthy tissues. ${ }^{16,22}$ In case of macroadenomas accompanied by severe displacement and compression of the normal pituitary glands, both sequences would measure the same bulk tumor, and regardless of resolution, the bulk tumor would be presumed to have the same mean ADC. When individual values were examined, the ADC measurements of the $2 \mathrm{DWI}$ sequences were also consistent. Furthermore, the present diffusion results revealed that the ADC of soft pituitary macroadenomas $\left(0.75 \pm 0.14 \times 10^{-3} \mathrm{~mm}^{2} / \mathrm{s}\right)$ was significantly lower than that of the anterior lobe of normal pituitary glands $\left(1.18 \pm 0.19 \times 10^{-3}\right.$ $\mathrm{mm}^{2} / \mathrm{s}$ ). To our knowledge, only 1 study to date has evaluated both pituitary adenomas and pituitary glands simultaneously; the authors reported no significant difference in ADC between pituitary adenomas and the unaffected anterior lobe of the pituitary glands. ${ }^{23}$ This discrepancy could be because the authors focused only on differences between different functional adenomas and pituitary glands, without considering tumor consistency. The present study included surgically and pathologically confirmed macroadenomas with soft consistency. Possible explanations for the low ADC of soft macroadenomas include an increase in cellularity, reduction in extracellular space, and the presence of cytoplasmic content with a relatively high nucleus-to-cytoplasm ratio. ${ }^{24}$ Besides, a pathologic study has proved that tumor cells form irregular sheets or masses and contain larger and more uniform nuclei than normal pituitary tissues. ${ }^{25}$ Moreover, low expression levels of laminin, fibronectin, reticulin, and type IV collagen in adenomatous pituitary tissues might also contribute to the low ADC. ${ }^{3,26}$ Our results appeared to be discordant with the study of Boxerman et al, ${ }^{3}$ who found that macrocystic and macrohemorrhagic macroadenomas and solid tumors with higher ADC were more likely to be successfully managed with an operation. This might be due to the differences in the proportion of tumor components in different study populations.

Because an endoscopic transsphenoidal technique is applied as a minimally invasive surgical method for resection of pituitary adenomas, tumor consistency is one of the most important parameters influencing the success of this approach. ${ }^{3}$ Soft pituitary macroadenomas account for most pituitary adenomas and are always associated with a low incidence of complications (CSF rhi- norrhea and hormonal deficiency). ${ }^{3,12}$ Resection of macroadenomas requires removal of the inferior or posterior portions first. ${ }^{12}$ Improved anatomic depiction of the sellar region with FOCUS DWI is most notable in regions near the skull base. Superior image quality is the fundamental basis of accurate disease assessment. Combined with the relationship of diffusivity between soft pituitary macroadenomas and normal pituitary glands, the FOCUS sequence provides a new perspective for evaluation of tumor consistency. It might help surgeons prepare detailed surgical strategies and decrease the rates of postoperative pain and discomfort.

This study has several limitations. First, the sample size was relatively small. Because we focused on only comparative qualitative and quantitative analysis of the 2 methods, we did not consider the functional variation of macroadenomas. Second, because pituitary macroadenomas with firm consistency occur in $4 \%-15 \%$ of patients, ${ }^{3,9}$ we encountered very few such cases. However, these patients were excluded because preoperative treatment might have induced fibrotic changes in tumors. Future studies would ideally comparatively evaluate the ADCs of adenomas of different consistencies and normal pituitary glands to achieve better results.

\section{CONCLUSIONS}

Relative to conventional single-shot EPI DWI, FOCUS DWI can acquire images of normal pituitary glands and pituitary macroadenomas with higher resolution and fewer susceptibility artifacts in a clinically feasible scan time. The FOCUS technique enables evaluation of the diffusivity of normal pituitary glands, which might serve as a reference for evaluating the consistency of macroadenomas in the future.

\section{REFERENCES}

1. Lomban E, Bonneville F, Karachi C, et al. Massive stroke in a patient with pituitary apoplexy, cervical carotid artery stenosis and hypotension. J Neuroradiol 2006;33:259-62 CrossRef Medline

2. Takayasu T, Yamasaki F, Tominaga A, et al. A pituitary abscess showing high signal intensity on diffusion-weighted imaging. Neurosurg Rev 2006;29:246-48 CrossRef Medline

3. Boxerman JL, Rogg JM, Donahue JE, et al. Preoperative MRI evaluation of pituitary macroadenoma: imaging features predictive of successful transsphenoidal surgery. AJR Am J Roentgenol 2010;195: 720-28 CrossRef Medline

4. Hiwatashi A, Yoshiura T, Togao O, et al. Evaluation of diffusivity in the anterior lobe of the pituitary gland: 3D turbo field echo with diffusion-sensitized driven-equilibrium preparation. AJNR Am J Neuroradiol 2014;35:95-98 CrossRef Medline

5. Mahmoud OM, Tominaga A, Amatya VJ, et al. Role of PROPELLER diffusion weighted imaging and apparent diffusion coefficient in the diagnosis of sellar and parasellar lesions. Eur J Radiol 2010;74: 420-27 CrossRef Medline

6. Le Bihan D, Poupon C, Amadon A, et al. Artifacts and pitfalls in diffusion MRI. J Magn Reson Imaging 2006;24:478-88 CrossRef Medline

7. Suzuki C, Maeda M, Hori K, et al. Apparent diffusion coefficient of pituitary macroadenoma evaluated with line-scan diffusionweighted imaging. J Neuroradiol 2007;34:228 -35 CrossRef Medline

8. Wei XE, Li WB, Li MH, et al. Detection of brain lesions at the skull base using diffusion-weighted imaging with readout-segmented echo-planar imaging and generalized autocalibrating partially parallel acquisitions. Neurol India 2011;59:839-43 CrossRef Medline

9. Yiping L, Ji X, Daoying G, et al. Prediction of the consistency of 
pituitary adenoma: a comparative study on diffusion-weighted imaging and pathological results. J Neuroradiol 2016;43:186-94 CrossRef Medline

10. Mahmoud OM, Tominaga A, Amatya VJ, et al. Role of PROPELLER diffusion-weighted imaging and apparent diffusion coefficient in the evaluation of pituitary adenomas. Eur J Radiol 2011;80:412-17 CrossRef Medline

11. Yiping L, Hui L, Kun Z, et al. Diffusion-weighted imaging of the sellar region: a comparison study of BLADE and single-shot echo planar imaging sequences. Eur J Radiol 2014;83:1239-44 CrossRef Medline

12. Theodros D, Patel M, Ruzevick J, et al. Pituitary adenomas: historical perspective, surgical management and future directions. CNS Oncol 2015;4:411-29 CrossRef Medline

13. Saritas EU, Cunningham CH, Lee JH, et al. DWI of the spinal cord with reduced FOV single-shot EPI. Magn Reson Med 2008;60: 468-73 CrossRef Medline

14. Vidiri A, Minosse S, Piludu F, et al. Feasibility study of reduced field of view diffusion-weighted magnetic resonance imaging in head and neck tumors. Acta Radiol 2017;58:292-300 CrossRef Medline

15. Ma C, Li YJ, Pan CS, et al. High resolution diffusion weighted magnetic resonance imaging of the pancreas using reduced field of view single-shot echo-planar imaging at 3 T. Magn Reson Imaging 2014; 32:125-31 CrossRef Medline

16. Dong H, Li Y, Li H, et al. Study of the reduced field-of-view diffusion-weighted imaging of the breast. Clin Breast Cancer 2014;14: 265-71 CrossRef Medline

17. Korn N, Kurhanewicz J, Banerjee S, et al. Reduced-FOV excitation decreases susceptibility artifact in diffusion-weighted MRI with endorectal coil for prostate cancer detection. Magn Reson Imaging 2015;33:56-62 CrossRef Medline

18. Riffel P, Michaely HJ, Morelli JN, et al. Zoomed EPI-DWI of the head and neck with two-dimensional, spatially-selective radiofrequency excitation pulses. Eur Radiol 2014;24:2507-12 CrossRef Medline

19. Pereira FP, Martins G, Figueiredo E, et al. Assessment of breast lesions with diffusion-weighted MRI: comparing the use of different b values. AJR Am J Roentgenol 2009;193:1030-35 CrossRef Medline

20. Mohamed FF, Abouhashem S. Diagnostic value of apparent diffusion coefficient (ADC) in assessment of pituitary macroadenoma consistency. The Egyptian Journal of Radiology and Nuclear Medicine 2013;44:617-24 CrossRef

21. Zaharchuk G, Saritas EU, Andre JB, et al. Reduced field-of-view diffusion imaging of the human spinal cord: comparison with conventional single-shot echo-planar imaging. AJNR Am J Neuroradiol 2011;32:813-20 CrossRef Medline

22. Singer L, Wilmes LJ, Saritas EU, et al. High-resolution diffusionweighted magnetic resonance imaging in patients with locally advanced breast cancer. Acad Radiol 2012;19:526-34 CrossRef Medline

23. Hiwatashi A, Togao O, Yamashita K, et al. Evaluation of diffusivity in pituitary adenoma: 3D turbo field echo with diffusion-sensitized driven-equilibrium preparation. British J Radiol 2016;89:20150755 CrossRef Medline

24. Pierallini A, Caramia F, Falcone C, et al. Pituitary macroadenomas: preoperative evaluation of consistency with diffusion-weighted MR imaging-initial experience. Radiology 2006;239:223-31 CrossRef Medline

25. Noh S, Kim SH, Cho NH, et al. Rapid reticulin fiber staining method is helpful for the diagnosis of pituitary adenoma in frozen section. Endocr Pathol 2015;26:178-84 CrossRef Medline

26. Jarzembowski J, Lloyd R, McKeever P. Type IV collagen immunostaining is a simple, reliable diagnostic tool for distinguishing between adenomatous and normal pituitary glands. Arch Pathol Lab Med 2007;131:931-35 Medline 